\title{
An IL-9 solution to inflammation resolution
}

adoptive
transfer of
ILC2s resolved
inflammation
in $I / 9^{-/-}$mice

Drugs for inflammation-driven diseases, such as rheumatoid arthritis, often aim to reduce the severity of the inflammatory response. Rauber et al. suggest an alternative strategy: promoting the resolution of inflammation. They found that IL-9 is needed for regulatory $\mathrm{T}\left(\mathrm{T}_{\text {reg }}\right)$ cells, which restore homeostasis following inflammation, and that IL-9 treatment attenuated inflammation in a mouse model of arthritis.

Increasing the resolution of inflammation is an attractive proposition, but therapies that aim to do so have not yet been successful, and little is known about the cytokines that govern this process. In this article, the authors found that mice deficient for IL-9 have a prolonged response to antigen-induced arthritis (AIA); joint swelling resolves spontaneously in 12-16 days in wild-type mice but persists beyond 42 days $I l 9^{-1-}$ mice without signs of resolution.

These $I l 9^{-/-}$mice had defects in $\mathrm{T}_{\text {reg }}$ cells. $\mathrm{T}_{\text {reg }}$ cells from $I l 9^{-/-}$mice with AIA had a reduced capacity to suppress effector $\mathrm{T}$ ( $\mathrm{T}_{\text {eff }}$ ) cells in co-cultures, providing a potential mechanism for the prolonged inflammation.

The authors found that the majority of IL-9-producing cells in AIA expressed markers of type 2 innate lymphoid cells (ILC2s). These cells are non-cytotoxic components of the innate immune system that promote anti-helminth immunity and tissue repair. Few ILC2s were found in inflamed joints in $I l^{-/-}$ mice, supporting previous reports that IL-9 supports ILC2
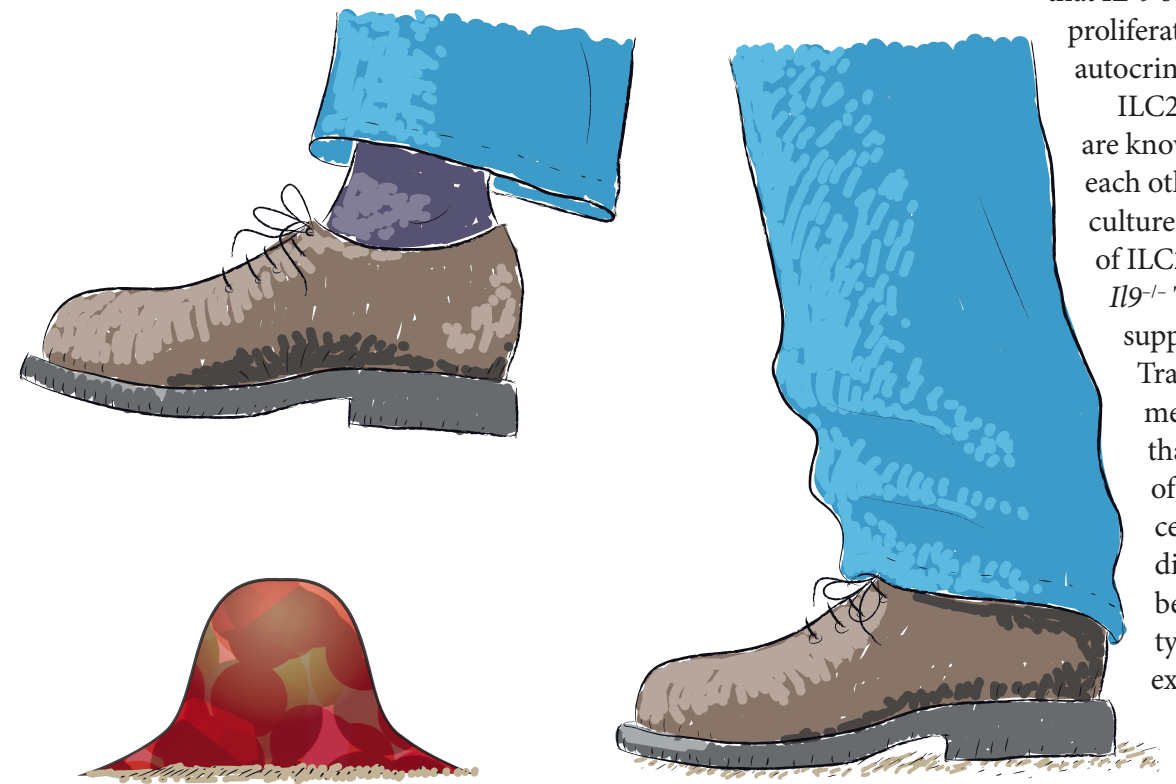
autocrine loop.

ILC2s and $\mathrm{T}_{\mathrm{reg}}$ cells are known to affect each other. In cell culture, the presence of ILC2s enabled Il9--- $\mathrm{T}_{\text {reg }}$ cells to suppress $\mathrm{T}_{\text {eff }}$ cells. Transwell experiments showed that the effect of ILC2s on $\mathrm{T}_{\text {reg }}$ cells required a direct interaction between these cell types. Subsequent experiments supported the hypothesis that IL-9 upregulates inducible T cell costimulator ligand (ICOSL) and glucocorticoid-induced tumour necrosis factor-related ligand (GITRL; also known as TNFSF18) on ILC2s, which bind to the receptors ICOS and GITR, respectively, on $\mathrm{T}_{\text {reg }}$ cells to promote $\mathrm{T}_{\text {reg }}$ cell activity. In the AIA model, adoptive transfer of GITR- and ICOS-activated $I l 9^{-1-} \mathrm{T}_{\text {reg }}$ cells back into $\mathrm{I} l 9^{-/-}$mice restored the kinetics of inflammation resolution. Similarly, adoptive transfer of ILC2s resolved inflammation in $I l 9^{-/-}$mice.

These findings are likely applicable to individuals with rheumatoid arthritis. Low numbers of IL- $9^{+}$ ILC2s were found in the synovial fluid samples of patients with active rheumatoid arthritis, whereas individuals in clinical remission had a high number of these cells.

Improving the resolution of inflammation is relevant to numerous immune disorders. In a mouse model of chronic arthritis, in which inflammation persists over several weeks, overexpression of IL-9 accelerated resolution, suggesting that IL-9 itself could be therapeutically useful. Such therapies would be unlikely to hamper the inflammatory response necessary to clear infections but would render chronic inflammation transient.

Megan Cully

ORIGINAL ARTICLE Rauber, S. et al. Resolution of inflammation by interleukin-9-producing type 2 innate lymphoid cells. Nat. Med. 23, 938-944 (2017) FURTHER READING Fullerton, J. N. \& Gilroy, D. W. Resolution of inflammation: a new therapeutic frontier. Nat. Rev. Drug Discov. 15, 551-567 (2016) 